\title{
Multiple Sclerosis Patients Treated With Diroximel Fumarate in the Real-World Setting Have High Rates of Persistence and Adherence
}

\author{
Jacob Liseno • Brittney Lager (D) C Catherine Miller (D) • \\ Sai L. Shankar · Jason P. Mendoza (D) · James B. Lewin (D)
}

Received: February 1, 2021 / Accepted: March 10, 2021 / Published online: April 12, 2021

(C) The Author(s) 2021

\begin{abstract}
Introduction: Persistence to multiple sclerosis (MS) disease-modifying therapy is fundamental for maximal treatment outcomes. Diroximel fumarate (DRF) is approved in the USA for relapsing MS. Following oral administration, DRF is metabolized to monomethyl fumarate, the active metabolite of dimethyl fumarate (DMF). DRF showed clinically significant improvements in gastrointestinal (GI) tolerability versus DMF in a head-to-head clinical trial; however, real-world persistence/adherence has not been assessed. We evaluated persistence/adherence in DRF-treated patients in a real-world clinical practice.

Methods: This retrospective analysis of the AcariaHealth Specialty Pharmacy Program included patients initiating DRF from 4 December 2019 through 3 April 2020 and followed until data extraction (31 August 2020). Exclusion criteria included undetermined treatment status (e.g., DRF prescription transfer to a different pharmacy). Endpoints included persistence (overall proportion of patients remaining on DRF), discontinuation rate due to
\end{abstract}

J. Liseno · B. Lager

AcariaHealth, Orlando, FL, USA

C. Miller · S. L. Shankar · J. P. Mendoza .

J. B. Lewin $(\square)$

Biogen, 225 Binney St, Cambridge, MA 02142, USA

e-mail: jim.lewin@biogen.com

GI adverse events (AEs), and adherence (proportion of days covered [PDC]). GI AEs included GI-related AEs occurring at any time, or any unknown $\mathrm{AE}$ without details about the nature of the event if the unknown $\mathrm{AE}$ occurred $\leq 90$ days after DRF initiation.

Results: Overall, 160 patients with MS were included. Median (range) patient age was 51 $(20-79)$ years, $80.6 \%(129 / 160)$ of patients were female, and $16.3 \%(26 / 160)$ had prior DMF treatment. Median (range) treatment duration was $7.6(0.1-10.4)$ months. Estimated proportion of patients remaining persistent on DRF treatment at 8 months was $88.6 \%$ (95\% confidence interval [CI] 82.5-2.7). Overall, 3.8\% (6/ 160) of patients discontinued due to GI AEs. Mean PDC was 91.4\% (95\% CI 89.1-93.7). In a DMF-to-DRF switch subgroup, 92.3\% (24/26) remained persistent on DRF, and 3.8\% (1/26) discontinued DRF due to GI AEs.

Conclusion: This real-world analysis of DRFtreated patients showed high overall persistence, low discontinuation rate due to GI AEs, and high adherence to therapy, aligning with expectations based on DRF clinical trials. Data were consistent in the DMF-to-DRF subgroup.

\section{Infographic:}




\section{Neurology and Therapy}

\section{Multiple Sclerosis Patients Treated With Diroximel Fumarate in the Real-World Setting Have High Rates of Persistence and Adherence}
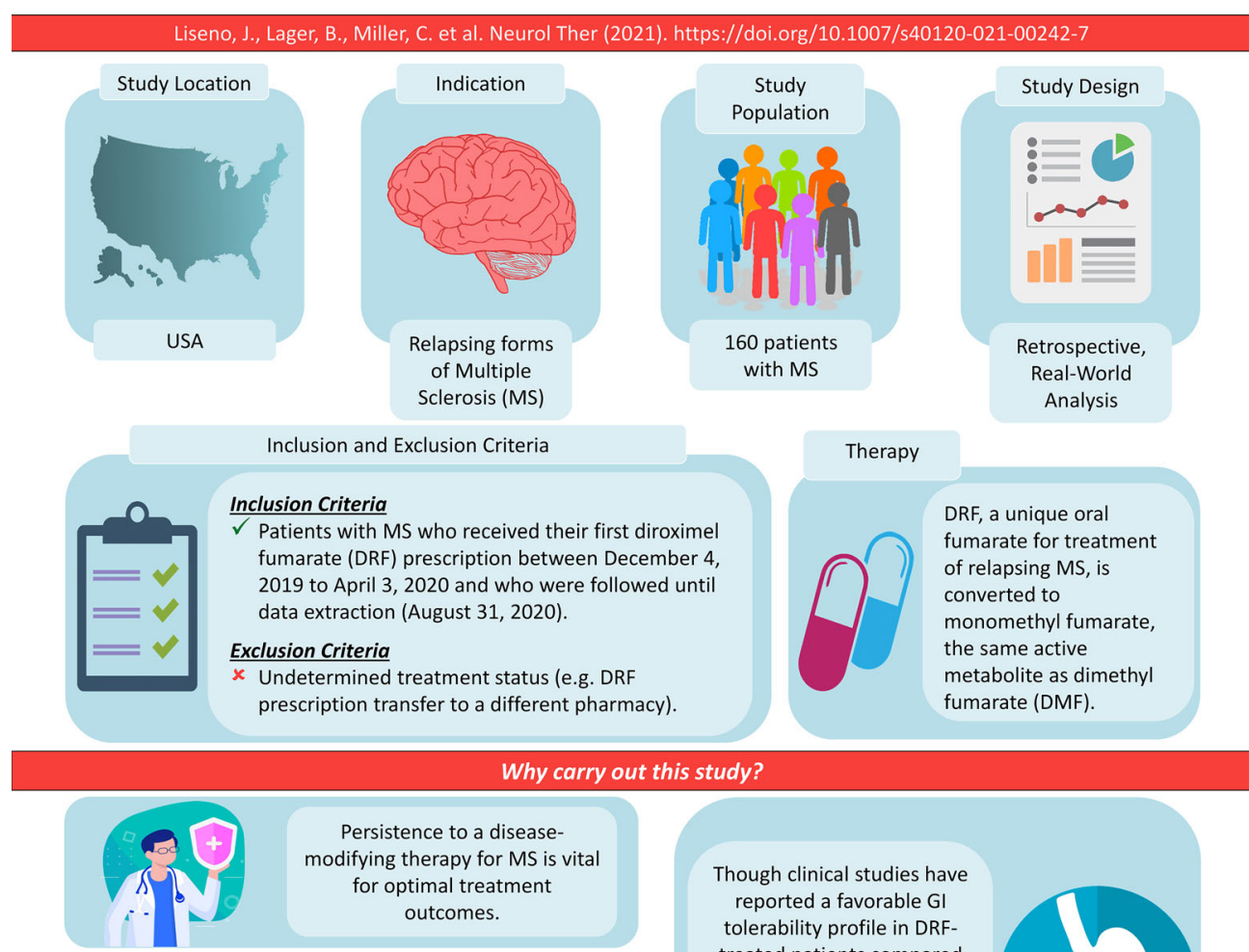

The objective of this study was to evaluate persistence to therapy,

discontinuation rates due to

gastrointestinal adverse events ( $\mathrm{Gl}$

$A E s)$, and adherence in patients

with MS treated with DRF in realworld clinical practice.

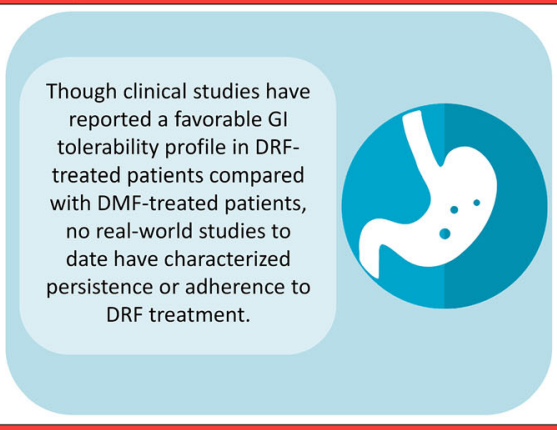

\section{What was learned from the study?}

The high level of both overall persistence (88.6\%) and adherence (mean PDC: $91.4 \%)$ reported in this first real-world analysis of DRF-treated patients further supports DRF as a valuable treatment option for patients with MS.

This analysis addresses a knowledge gap in the practice of neurology regarding persistence, adherence, and treatment discontinuation due to GI AEs in patients with MS treated with DRF in real-world clinical practice.
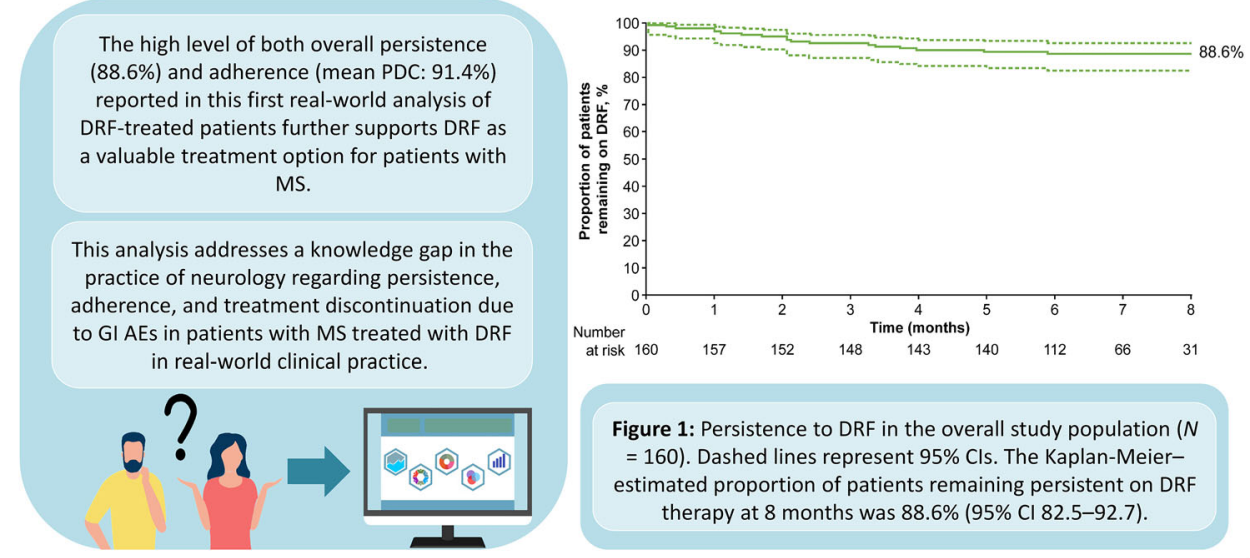

Figure 1: Persistence to DRF in the overall study population ( $N$ $=160$ ). Dashed lines represent 95\% Cls. The Kaplan-Meierestimated proportion of patients remaining persistent on DRF therapy at 8 months was $88.6 \%$ (95\% Cl 82.5-92.7). 
Keywords: Adherence; Dimethyl fumarate; Diroximel fumarate; Gastrointestinal side effects; Multiple sclerosis; Real-world treatment; Tolerability

\section{DIGITAL FEATURES}

This article is published with digital features, including a summary slide and infographic, to facilitate understanding of the article. To view digital features for this article go to https://doi. org/10.6084/m9.figshare.14179409.

\section{INTRODUCTION}

Many disease-modifying therapies (DMTs) have been introduced to the market over the last 20 years for the treatment of multiple sclerosis (MS) [1], with nearly 20 oral, injectable, and infused medications now available [2, 3]. Persistence to DMT treatment enables patients to achieve optimal results from their medication in chronic diseases such as MS [4, 5]. With numerous treatment options now available for MS, it is important that the chosen DMT not only has a benefit-risk profile appropriate for the patient's MS, but is also a treatment the patient can tolerate [6, 7]. Factors in patient treatment preferences may include anticipated gains in efficacy, frequency and mode of administration, and side effects [7].

Diroximel fumarate (DRF) is an oral fumarate approved in the USA for the treatment of relapsing forms of MS, administered as a maintenance dose of two 231-mg capsules twice daily [8]. Following oral administration, DRF is metabolized to monomethyl fumarate, the same pharmacologically active metabolite as dimethyl fumarate (DMF) and, therefore, the overall safety and efficacy profiles of DRF and DMF are expected to be similar [8, 9]. DMF has demonstrated a favorable benefit-risk profile in clinical studies of patients with relapsing-remitting MS; however, some patients taking DMF experience gastrointestinal (GI) adverse events (AEs) early in treatment that may lead to dose interruption or discontinuation [10-12]. Compared with DMF, DRF has a distinct chemical structure and a higher molecular weight that is hypothesized to produce less GI irritation in the GI tract and improve GI tolerability due to less reactivity with off-target proteins [13].

Two phase 3 clinical trials of DRF have demonstrated favorable GI tolerability and low rates of GI-related treatment discontinuation in patients with relapsing-remitting MS. DRF demonstrated clinically significant improvements in GI tolerability compared with DMF in the phase 3, randomized, head-to-head EVOLVE-MS-2 study, with significantly fewer days of patient-assessed GI symptoms and lower rates of GI AEs (DRF 34.8\% vs. DMF 49.0\%) and discontinuation due to GI AEs (DRF $0.8 \%$ vs. DMF 4.8\%) [14]. DRF demonstrated a similarly low rate of treatment discontinuation due to GI AEs (0.7\%) in the ongoing, 2-year, single-arm, open-label EVOLVE-MS-1 study [15]. Adherence to DRF was also high in EVOLVE-MS-1, with a mean treatment adherence of 93\% [16] and, importantly, $91 \%$ of patients were $\geq 80 \%$ adherent to treatment [16]. A post hoc analysis of EVOLVE-MS-2 showed that the improved GI profile of DRF compared with DMF translated into clinically meaningful benefits to quality of life and work productivity [17].

The rate of treatment discontinuation observed in clinical trials may not be indicative of the rate observed in real-world clinical practice. This is not unexpected given the stringent inclusion criteria used in phase 3 clinical trials compared with the more diverse patient population receiving treatment in a real-world setting, as well as the structured nature of the phase 3 clinical trial setting. In studies with DMF, the rate of treatment discontinuation due to GI AEs was approximately $4 \%$ in randomized phase 3 clinical trials [9], whereas the rate was significantly higher in real-world studies, varying from 5 to $19 \%$ in studies ranging in duration from 3 to 37 months [18-23].

Although the observed rate of treatment discontinuation due to GI AEs was $<1 \%$ with DRF in clinical trials, there are no data characterizing GI-related discontinuations or persistence to treatment in patients treated with DRF in the real-world setting. Based on DMF experience, we expect to see an increase in real- 
world DRF discontinuation rates relative to clinical studies. We also expect that adherence to DRF in the real-world setting might not be as high as that observed in clinical trials, based on data suggesting that average adherence to MS DMTs may be approximately 60\% [24], and previous studies have shown DMF to have a mean adherence rate of approximately $70 \%$ $[24,25]$. Therefore, the objective of this study was to evaluate persistence to therapy, discontinuation rates due to GI AEs, and adherence in patients with MS treated with DRF in real-world clinical practice using the AcariaHealth Specialty Pharmacy Program (SPP).

\section{METHODS}

\section{Data Source}

A retrospective analysis of the AcariaHealth SPP included patients with MS who received their first DRF prescription from the specialty pharmacy provider AcariaHealth (Troy, MI, USA) from 4 December 2019 through 3 April 2020. Patients were followed until data extraction on 31 August 2020. Based on the dates of DRF initiation and data extraction, all patients in the study had the potential to be treated with DRF for nearly 5 months. Patients were excluded if their treatment status was indeterminate, such as in the case of transferral of the DRF prescription to a different pharmacy. Information on a patient's prior DMT use was based on pharmacy records.

This was a retrospective noninterventional study. All patient information was anonymized, and patient confidentiality was maintained through compliance with Health Insurance Portability and Accountability Act (HIPAA) regulations. This analysis is based on previously collected data and does not involve any new studies of human or animal subjects performed by any of the authors.

\section{Study Endpoints}

Endpoints included persistence (defined as the overall proportion of patients remaining on therapy), discontinuation rate due to GI AEs, and adherence. GI AEs included events that were directly GI related, in addition to any unknown $\mathrm{AE}$ (i.e., an $\mathrm{AE}$ lacking details regarding the nature of the event) that occurred within 90 days of initiating DRF therapy. We undertook this analytical approach to avoid underestimation of the GI AE discontinuation rate. Information on patient AEs and reasons for treatment discontinuation were collected by AcariaHealth pharmacy staff prior to each prescription refill and recorded in the pharmacy database. Adherence, as measured by the proportion of days covered (PDC), was calculated as the number of days that a patient is "covered" by having medication on hand in the treatment period, divided by the total number of days in the treatment period, multiplied by $100 \%$.

\section{Statistical Analysis}

Descriptive statistics were used to summarize demographic and clinical characteristics of the study population. Continuous variables were summarized using the mean (standard deviation [SD]) or median (range) as appropriate, and categorical variables were summarized using frequency (percentage). Persistence was characterized using the Kaplan-Meier method with 95\% confidence intervals (CIs). Although some patients were treated for up to 10 months, the Kaplan-Meier estimate for DRF persistence was reported for up to 8 months to ensure a meaningful sample size. Discontinuation rate and PDC were also characterized with 95\% CIs.

To account for a situation in which a healthcare provider (HCP) prescribed an extended titration period beyond the US prescribing information-recommended 1-week titration for DRF, potentially causing a gap or delay for obtaining the first refill, we conducted a PDC sensitivity analysis, in which we excluded month 1 (the first DRF shipment) from the PDC calculation. We also evaluated PDC in a subgroup of patients who were treated with DRF for $\geq 6$ months to determine whether the PDC could be impacted by patients who have only been on treatment for $<6$ months. Finally, we assessed discontinuation rate and 
Table 1 Patient demographics and baseline disease characteristics: overall study population

Age, years

$$
\begin{aligned}
& \text { Median (range) } \\
& \text { Age }<55 \text { years } \\
& \text { Age } \geq 55 \text { years }
\end{aligned}
$$$$
51(20-79)
$$$$
100(62.5)
$$

Female

MS diagnosis

Confirmed by ICD-10 code for MS

Inferred by drug therapy classification of MS

US region ${ }^{\mathrm{a}}$

Northeast

Midwest

South

West

No prior $\mathrm{DMT}^{\mathrm{b}}$

Prior DMT

Interferon

Teriflunomide

Dimethyl fumarate

DRF treatment duration, months, median (range)

$7.6(0.1-10.4)$

All values reported as $n(\%)$ unless otherwise indicated

$D M T$ Disease-modifying therapy, $D R F$ diroximel fumarate, $I C D-10$ International Statistical Classification of Diseases and Related Health Problems, Tenth Revision, $M S$ multiple sclerosis

a Regional breakdown based on 2020 US Census categories for region

b Based on pharmacy record

discontinuation due to GI AEs in a subgroup of patients who had received DMF as their most recent DMT before switching to DRF.

\section{RESULTS}

\section{Overall Study Population}

Overall, the analysis included 160 patients with MS; the median (range) age was 51 (20-79) years, and $80.6 \%(129 / 160)$ of patients were women (Table 1). A total of $16.3 \%(26 / 160)$ of patients had received prior DMF treatment. Median (range) DRF treatment duration was 7.6 (0.1-10.4) months.

The estimated proportion of patients remaining persistent on DRF treatment at 8 months was $88.6 \% \quad(95 \%$ CI $82.5-92.7)$ (Fig. 1). Overall, 3.8\% (95\% CI 1.4-8.2) of patients discontinued due to GI AEs (Table 2). Mean PDC was 91.4\% (95\% CI 89.1-93.7) overall and remained high, both when excluding the first DRF fill to account for extended titration (94.6\%; 95\% CI 93.4-95.9) and in a subgroup of patients treated for $\geq 6$ months 


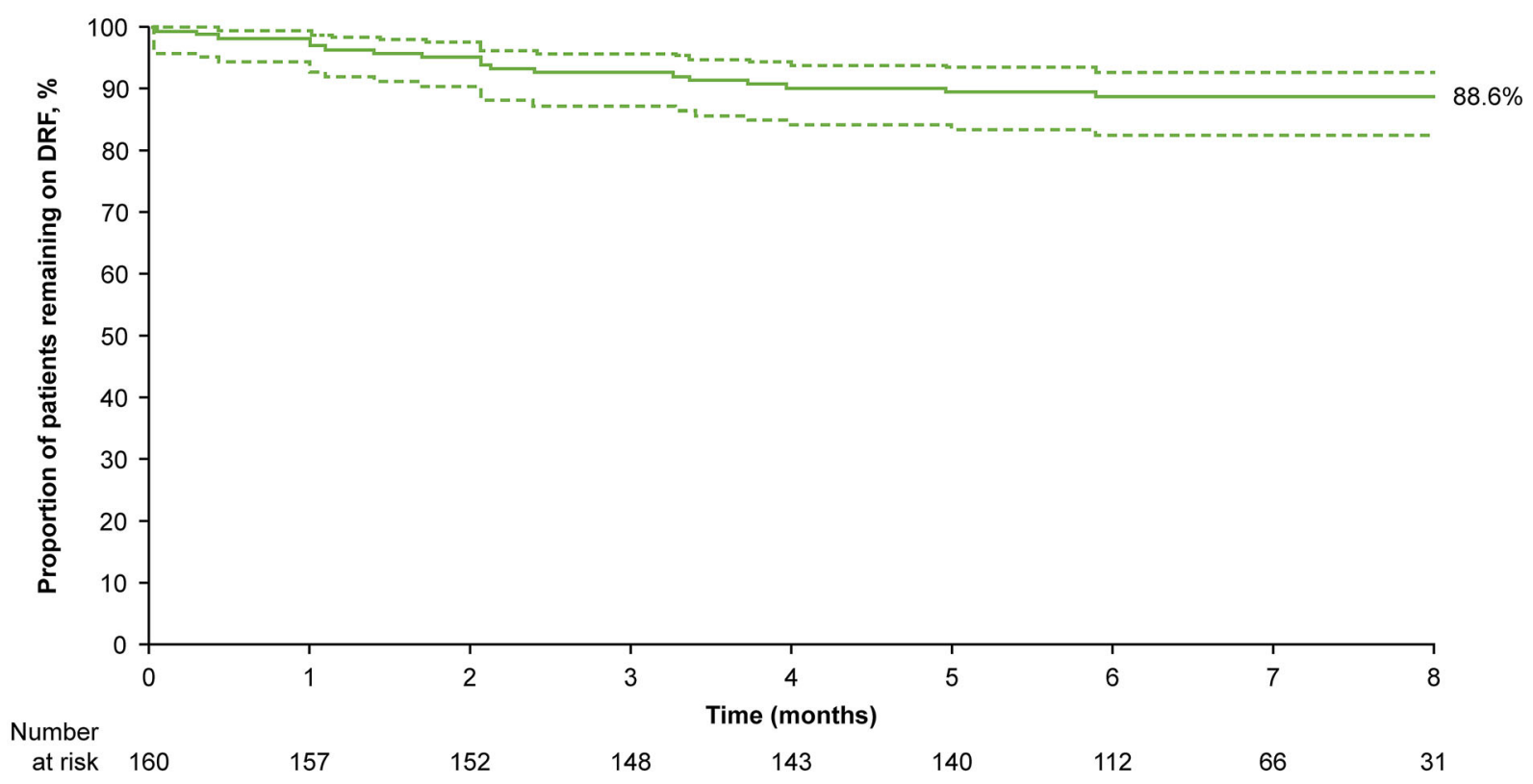

Fig. 1 Persistence to DRF in the overall study population $(N=160)$. Dashed lines represent 95\% confidence intervals. The Kaplan-Meier-estimated proportion of patients remaining persistent on DRF therapy at 8 months was 88.6\% (95\% CI 82.5-92.7). DRF Diroximel fumarate

Table 2 Treatment discontinuation rate and discontinuation due to gastrointestinal adverse events

\begin{tabular}{|c|c|c|c|c|}
\hline \multirow[t]{2}{*}{ Characteristic } & \multicolumn{2}{|c|}{ Overall $(N=160)$} & \multicolumn{2}{|c|}{ DMF-to-DRF subgroup $(n=26)$} \\
\hline & $\overline{n(\%)}$ & $95 \% \mathrm{CI}$ & $n(\%)$ & $95 \% \mathrm{CI}$ \\
\hline Discontinued DRF & $18(11.3)$ & $6.7-17.8$ & $2(7.7)$ & $0.9-27.8$ \\
\hline Discontinued DRF due to GI AE & $6^{\mathrm{a}}(3.8)$ & $1.4-8.2$ & $1^{\mathrm{b}, \mathrm{c}}(3.8)$ & $0.1-21.4$ \\
\hline
\end{tabular}

$A E$ adverse event, $D M F$ dimethyl fumarate, $G I$ gastrointestinal

a In the overall population, the other non-GI related reason for DRF treatment discontinuation included "other AE" $(n=11)$ and "physician decision-pursuing alternate therapy" $(n=1)$

b The one patient in the DMF-to-DRF subgroup who discontinued DRF due to GI AEs had also discontinued prior DMF therapy due to GI AE at 60 days after DMF initiation. After switching to DRF, the patient was treated for 30 days prior to discontinuing treatment

c In the DMF-to-DRF subgroup, the other non-GI related reasons for DRF treatment discontinuation included "other AE" $(n=1)$

(92.3\%; 95\% CI 87.3-97.2) (Fig. 2a). The proportion of patients with PDC $\geq 80 \%$ was $88.1 \%$ overall, 93.6\% when excluding the first DRF fill, and $87.5 \%$ in patients treated for $\geq 6$ months (Fig. 2b).

\section{DMF-to-DRF Subgroup}

Demographics of the 26 patients within the DMF-to-DRF subgroup are shown in Table 3. Median (range) age (51 [35-79] years) and proportion of female patients $(84.6 \%$ [22/26]) were similar to the overall study population. Fifteen of the patients in this subgroup had a known reason for discontinuing prior DMF therapy; the 
(a) Mean PDC

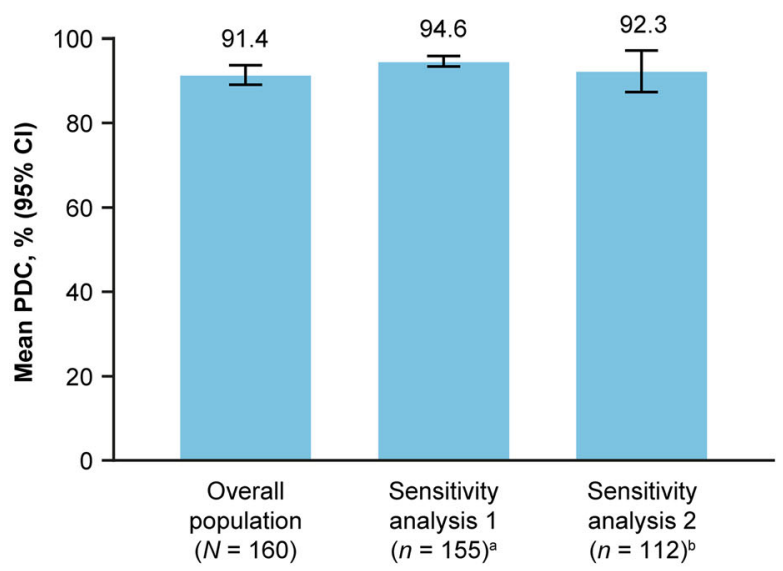

Fig. 2 Adherence to DRF in the overall study population. a Mean PDC, $\mathbf{b}$ proportion of patients with PDC $\geq 80 \%$. ${ }^{a}$ Sensitivity analysis 1: excluding first DRF fill to account for healthcare provider-prescribed extended titration

remaining 11 discontinued DMF for unknown reasons. Of the 15 patients with a known reason for discontinuing prior DMF therapy, $86.7 \%$ $(13 / 15)$ had switched to DRF due to GI tolerability issues while on DMF treatment, and $13.3 \%(2 / 15)$ discontinued prior DMF therapy due to an AE unrelated to GI AE issues (acne $n=1$, flushing/flushing-related events $n=1$ ). Median (range) duration of prior DMF therapy in the DMF-to-DRF subgroup was $7.2(0.2-56.5)$ months; however, median (range) duration of prior DMF therapy in the 13 patients who discontinued DMF due to GI AEs was 2.0 (0.2-10.9) months. Median (range) DRF treatment duration in the DMF-to-DRF switch subgroup was 6.8 (1.0-8.4) months.

In the DMF-to-DRF subgroup, 92.3\% (24/26) of patients remained persistent on DRF at the time of the analysis. The rate of DRF treatment discontinuation due to GI AEs was 3.8\% (1/26). The one patient who discontinued due to a GI AE had also discontinued prior DMF therapy due to a GI AE 60 days after DMF initiation. After switching to DRF, the patient was treated for 30 days before discontinuing therapy. Aside from this one patient, none of the other DMFto-DRF switch patients discontinued DRF due to GI AEs. (b) Proportion of patients with PDC $\geq 80 \%$

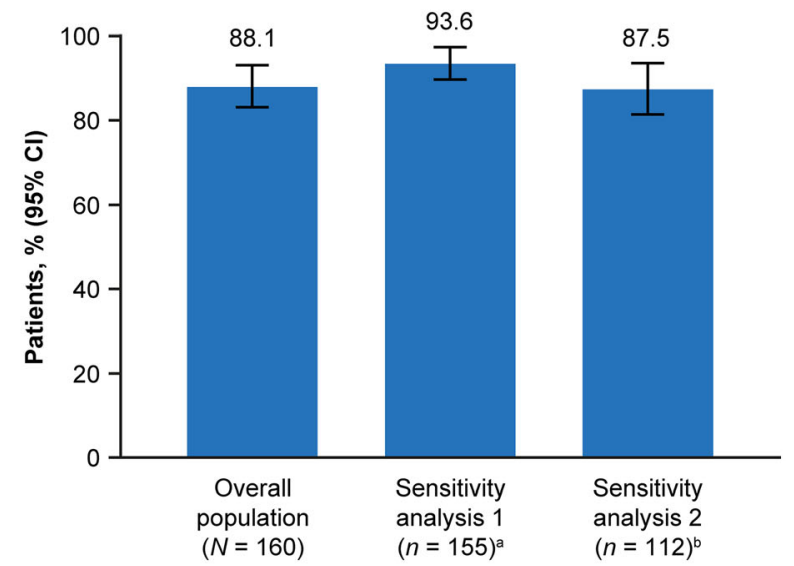

regimens. ${ }^{b}$ Sensitivity analysis 2 : subgroup of patients with $\geq 6$ months treatment duration. $D R F$ diroximel fumarate, $C I$ Confidence interval, $P D C$ proportion of days covered

\section{DISCUSSION}

In this real-world analysis of 160 patients with MS treated with DRF, overall persistence was high, discontinuation rate due to GI AEs was low, and patients were highly adherent to therapy, consistent with expectations based on the GI tolerability profile shown in clinical trials [14-16]. When DMF moved from clinical trials to real-world practice, there was a three- to fourfold increase in GI-related discontinuation rates. The DRF GI discontinuation rate in this study $(3.8 \%)$ is higher than the $<1 \%$ rate seen in clinical trials $[14,15]$, but it is still lower than the observed rate of GI discontinuations with DMF (5-19\%) based on previous real-world studies spanning 3 months to 3 years [18-23].

The DRF adherence rate in this analysis was considerably higher than rates previously observed with other DMTs, including DMF. DRF-treated patients in this study had an overall mean PDC of $91.4 \%$, while data from a large claims database found that treatment adherence in the year after DMT initiation was $69 \%$ with DMF, $63 \%$ with interferon beta or glatiramer acetate, $67 \%$ with teriflunomide, and $79 \%$ with fingolimod [25]. The high rate of adherence with DRF indicated that gaps in therapy fulfillment were infrequent. Some HCPs 
Table 3 Patient demographics and baseline disease characteristics: DMF-to-DRF subgroup

Patient demographics and baseline characteristics

DMF-to-DRF subgroup $(N=26)$

Age, years
Median (range)
$51(35-79)$
Age $<55$ years
$14(53.8)$
Age $\geq 55$ years
$12(46.2)$
Female
$22(84.6)$
MS diagnosis
Confirmed by ICD-10 code for MS
$22(84.6)$
Inferred by drug therapy classification of MS
$4(15.4)$
US region ${ }^{\mathrm{a}}$
Northeast
Midwest
South
West
Reason for discontinuing prior DMF therapy
Known reason
GI AE
Other $\mathrm{AE}^{\mathrm{b}}$
Unknown reason
Prior DMF treatment duration, months, median (range)
$7.2(0.2-56.5)$
DRF treatment duration, months, median (range)
$6.8(1.0-8.4)$

All values reported as $n$ with the percentage in parenthesis, unless otherwise indicated

$A E$ adverse event, $D R F$ diroximel fumarate, GI gastrointestinal, ICD-10 International Statistical Classification of Diseases and Related Health Problems, Tenth Revision, $M S$ multiple sclerosis

a Regional breakdown based on 2020 US Census categories for region

b One patient reported acne as the AE leading to discontinuation, and 1 patient reported flushing/flushing-related events

may prescribe an extended titration regimen with fumarates at initiation to preemptively address potential tolerability [26]; under these circumstances, a patient may be delayed in requesting their first refill. When accounting for this in the sensitivity analysis by excluding the first prescription from the PDC calculation, we did observe an increase in mean PDC to $94.6 \%$. PDC was also high in patients with a DRF treatment duration of $\geq 6$ months, suggesting that the two-capsule, twice-daily dosing regimen did not impact a patient's ability to maintain dosing as prescribed. Irrespective of analytical method, these data suggest a high level of adherence in this DRF-treated patient population. Previous studies have shown that patients who were more adherent to MS therapy were at a lower risk of relapse and MS-related inpatient hospitalizations, had lower MS-related costs, and had fewer ambulatory care visits or physician visits [27-29].

In the DMF-to-DRF subgroup, one-half (13/ 26) of patients had switched to DRF owing to GI tolerability issues on DMF, although it is 
possible that the number may be underestimated, as an additional 11 (42.3\%) patients had an unknown reason for discontinuing treatment with DMF before starting DRF. Similar to the overall patient group, the GI AE discontinuation rate was low (3.8\%) in the DMF-to-DRF switch subgroup, but the sample size of patients previously treated with DMF was small and additional follow-up is therefore warranted.

Consensus on what is deemed an acceptable level of adherence is yet to be reached [30], although a PDC of $\geq 80 \%$ is considered to be adherent in many disease states, including MS [31-34]. For MS, past research has shown that patients on DMTs with a PDC of $\geq 80 \%$ have better treatment outcomes [35]. Further, the US government's Centers for Medicare and Medicaid Services Star Rating system for pharmacy quality measures specifies that prescriptions for statins, noninsulin diabetes (oral antiglycemic agents), and renin angiotensin system antagonists should cover $\geq 80 \%$ of days in a period in order to be deemed adherent [36]. Adherence to DRF in our study population was high. Here, we report that the overall proportion of patients with $\mathrm{PDC} \geq 80 \%$ was $88.1 \%$ in the overall population, $87.5 \%$ in patients treated for $\geq 6$ months, and $93.6 \%$ when excluding the first DRF fill.

There were limitations to this study. While it would be desirable to further our research with comparative analyses versus other DMTs, the AcariaHealth SPP lacks detailed baseline characteristics available through other types of data sources, such as a retrospective chart review, limiting its use for comparative studies that require extensive baseline data to ensure balanced comparator groups. Nevertheless, the AcariaHealth SPP is a valuable data source for characterization of early real-world experience with DRF. In addition, PDC measures timely refilling and whether the patient has access to the drug, but it cannot definitively determine if a patient is taking each dose of medication as directed. However, we feel confident that the PDC data provide a reasonable estimate of adherence because patients in this study had to indicate when the next refill was required due to AcariaHealth's policy of not automatically shipping DRF refills to patients. We elected to use PDC to measure adherence in this study rather than medication possession ratio, which is an alternative measure that represents the sum of days' supply for all prescription fills relative to the number of days in the treatment period. We chose PDC because the medication possession ratio could be $>100 \%$ if a patient obtains medication earlier than required (i.e., early refills). The PDC ratio provides a more accurate representation of medication adherence by eliminating the possibility of being unreasonably elevated. Despite some limitations, PDC is widely accepted as a valid measure of patient adherence and is the preferred method for assessing adherence by the Pharmacy Quality Alliance for use in the Medicare plan Star Ratings [36]. A further limitation of this study is the short follow-up period. Although the median DRF treatment duration in the current analysis was only 7.6 months, we believe it is clinically relevant based on previous studies of DMF and DRF showing that GI tolerability issues generally occur early in treatment, typically within 3 months of DMF or DRF initiation $[12,15]$. Our data characterize the initial, short-term, real-world experience with DRF; additional follow-up is warranted to evaluate longer-term persistence and adherence to DRF.

\section{CONCLUSIONS}

In conclusion, this real-world analysis of DRFtreated patients showed that overall persistence was high (estimated proportion remaining persistent at 8 months $88.6 \%$, (95\% CI 82.5-92.7), discontinuation rate due to GI AEs was low $(3.8 \%)$, and patients were highly adherent to therapy (mean PDC 91.4\%), consistent with expectations based on clinical trials. Similar results were observed in patients who switched from DMF to DRF treatment. Future analyses of the AcariaHealth SPP with larger patient numbers and longer follow-up will provide additional insights on persistence and adherence to DRF in the real-world setting. 


\section{ACKNOWLEDGEMENTS}

Funding. This study was funded by Biogen (Cambridge, MA, USA). Biogen also provided funding for the journal's Rapid Service Fee.

Medical Writing, Editorial, and Other Assistance. Biogen provided funding for medical writing and editorial support in the development of this manuscript; Suzanne Douthwaite, CMPP (Excel Medical Affairs), wrote the first draft of the manuscript based on input from authors, and Kristen DeYoung (Excel Medical Affairs) copyedited and styled the manuscript per journal requirements.

Authorship. All named authors meet the International Committee of Medical Journal Editors (ICMJE) criteria for authorship for this article, take responsibility for the integrity of the work as a whole, and have given their approval for this version to be published.

Authors' Contributions. All named authors contributed to the design of the study. Data collection was performed by JL and BL. Data analysis and interpretation was performed by all authors. JL performed the statistical analyses. All authors participated in drafting/critically revising the manuscript.

Disclosures. Jacob Liseno and Brittney Lager are employees of AcariaHealth. Catherine Miller, Sai L. Shankar, Jason P. Mendoza, and James B. Lewin are employees of and hold stock/stock options in Biogen.

Compliance with Ethics Guidelines. All patient information was anonymized, and patient confidentiality was maintained through compliance with Health Insurance Portability and Accountability Act (HIPAA) regulations. This analysis is based on previously collected data and does not involve any new studies of human or animal subjects performed by any of the authors.

Data Availability. The datasets generated during and/or analyzed during the current study are available from the corresponding author on reasonable request.

Open Access. This article is licensed under a Creative Commons Attribution-NonCommercial 4.0 International License, which permits any non-commercial use, sharing, adaptation, distribution and reproduction in any medium or format, as long as you give appropriate credit to the original author(s) and the source, provide a link to the Creative Commons licence, and indicate if changes were made. The images or other third party material in this article are included in the article's Creative Commons licence, unless indicated otherwise in a credit line to the material. If material is not included in the article's Creative Commons licence and your intended use is not permitted by statutory regulation or exceeds the permitted use, you will need to obtain permission directly from the copyright holder. To view a copy of this licence, visit http:// creativecommons.org/licenses/by-nc/4.0/.

\section{REFERENCES}

1. Pardo G, Jones DE. The sequence of diseasemodifying therapies in relapsing multiple sclerosis: safety and immunologic considerations. J Neurol. 2017;264:2351-74.

2. Doshi A, Chataway J. Multiple sclerosis, a treatable disease. Clin Med (Lond). 2016;16:s53-9.

3. National MS Society. Disease-modifying therapies for MS. https://www.nationalmssociety.org/ NationalMSSociety/media/MSNationalFiles/ Brochures/Brochure-The-MS-Disease-ModifyingMedications.pdf. Accessed 5 Jan 2021.

4. Bandari DS, Sternaman D, Chan T, Prostko CR, Sapir T. Evaluating risks, costs, and benefits of new and emerging therapies to optimize outcomes in multiple sclerosis. J Manag Care Pharm. 2012;18: $1-17$.

5. Lizán L, Comellas M, Paz S, Poveda JL, Meletiche DM, Polanco C. Treatment adherence and other patient-reported outcomes as cost determinants in multiple sclerosis: a review of the literature. Patient Prefer Adherence. 2014;8:1653-64. 
6. Cree BAC, Mares J, Hartung H-P. Current therapeutic landscape in multiple sclerosis: an evolving treatment paradigm. Curr Opin Neurol. 2019;32: 365-77.

7. Mortensen GL, Rasmussen PV. The impact of quality of life on treatment preferences in multiple sclerosis patients. Patient Prefer Adherence. 2017;11:1789-96.

8. Biogen Inc. VUMERITY ${ }^{\circledR}$ (diroximel fumarate). Prescribing Information. 2020. Cambridge: Biogen Inc. Accessed 28 Oct 2020.

9. Biogen Inc. TECFIDERA ${ }^{\circledR}$ (dimethyl fumarate). Prescribing information. 2020. Cambridge: Biogen Inc. Accessed 28 Oct 2020.

10. Fox RJ, Miller DH, Phillips JT, et al. Placebocontrolled phase 3 study of oral BG-12 or glatiramer in multiple sclerosis. N Engl J Med. 2012;367: 1087-97.

11. Gold R, Kappos L, Arnold DL, et al. Placebocontrolled phase 3 study of oral BG-12 for relapsing multiple sclerosis. N Engl J Med. 2012;367: 1098-107.

12. Phillips JT, Selmaj K, Gold R, et al. Clinical significance of gastrointestinal and flushing events in patients with multiple sclerosis treated with delayed-release dimethyl fumarate. Int J MS Care. 2015;17:236-43.

13. Palte MJ, Wehr A, Tawa $M$, et al. Improving the gastrointestinal tolerability of fumaric acid esters: early findings on gastrointestinal events with diroximel fumarate in patients with relapsingremitting multiple sclerosis from the phase 3, openlabel EVOLVE-MS-1 study. Adv Ther. 2019;36: 3154-65.

14. Naismith RT, Wundes A, Ziemssen T, EVOLVE-MS-2 Study Group, et al. Diroximel fumarate demonstrates an improved gastrointestinal tolerability profile compared with dimethyl fumarate in patients with relapsing-remitting multiple sclerosis: results from the randomized, double-blind, phase III EVOLVE-MS-2 study. CNS Drugs. 2020;34: 185-96.

15. Naismith RT, Wolinsky JS, Wundes A, et al. Diroximel fumarate (DRF) in patients with relapsingremitting multiple sclerosis: interim safety and efficacy results from the phase 3 EVOLVE-MS-1 study. Mult Scler. 2020;26:1729-39.

16. Fink MK, Jasinska E, Repovic P, et al. High rates of adherence to oral diroximel fumarate are observed and sustained in patients with RMS. Int J MS Care. 2020;22(Suppl 2):34.
17. Wundes A, Wray S, Gold, R, et al. Improved gastrointestinal profile with diroximel fumarate is associated with a positive impact on quality of life compared with dimethyl fumarate: results from the randomized, double-blind, phase III EVOLVE-MS-2 study. Ther Adv Neurol Disord. 2021;14:1-14. https://doi.org/10.1177/1756286421993999

18. Fox EJ, Vasquez A, Grainger W, et al. Gastrointestinal tolerability of delayed-release dimethyl fumarate in a multicenter, open-label study of patients with relapsing forms of multiple sclerosis (MANAGE). Int J MS Care. 2016;18:9-18.

19. Vollmer B, Nair KV, Sillau SH, Corboy J, Vollmer T, Alvarez E. Comparison of fingolimod and dimethyl fumarate in the treatment of multiple sclerosis: two-year experience. Mult Scler J Exp Transl Clin. 2017;3:2055217317725102.

20. Chinea A, Amezcua L, Vargas W, et al. Real-world safety and effectiveness of dimethyl fumarate in Hispanic or Latino patients with multiple sclerosis: 3-year results from ESTEEM. Neurol Ther. 2020;9: 495-504.

21. Williams MJ, Amezcua L, Okai A, et al. Real-world safety and effectiveness of dimethyl fumarate in black or African American patients with multiple sclerosis: 3-year results from ESTEEM. Neurol Ther. 2020;9:483-93.

22. Kresa-Reahl K, Repovic P, Robertson D, Okwuokenye M, Meltzer L, Mendoza JP. Effectiveness of delayed-release dimethyl fumarate on clinical and patient-reported outcomes in patients with relapsing multiple sclerosis switching from glatiramer acetate: RESPOND, a prospective observational study. Clin Ther. 2018;40:2077-87.

23. Min J, Cohan S, Alvarez E, et al. Real-world characterization of dimethyl fumarate-related gastrointestinal events in multiple sclerosis: management strategies to improve persistence on treatment and patient outcomes. Neurol Ther. 2019;8:109-19.

24. Johnson KM, Zhou H, Lin F, Ko JJ, Herrera V. Realworld adherence and persistence to oral diseasemodifying therapies in multiple sclerosis patients over 1 year. J Manag Care Spec Pharm. 2017;23: 844-52.

25. Boster A, Nicholas J, Wu N, et al. Real-world comparison of relapse rates in patients with multiple sclerosis treated with disease-modifying therapies. Int J MS Care. 2016;18(Suppl 1):50.

26. Phillips JT, Agrella S, Fox RJ. Dimethyl fumarate: a review of efficacy and practical management strategies for common adverse events in patients with multiple sclerosis. Int J MS Care. 2017;19: 74-83. 
27. Gerber B, Cowling T, Chen G, Yeung M, Duquette $P$, Haddad P. The impact of treatment adherence on clinical and economic outcomes in multiple sclerosis: real world evidence from Alberta, Canada. Mult Scler Relat Disord. 2017;18:218-24.

28. Menzin J, Caon C, Nichols C, White LA, Friedman M, Pill MW. Narrative review of the literature on adherence to disease-modifying therapies among patients with multiple sclerosis. J Manag Care Pharm. 2013;19:S24-40.

29. Tan H, Cai Q, Agarwal S, Stephenson JJ, Kamat S. Impact of adherence to disease-modifying therapies on clinical and economic outcomes among patients with multiple sclerosis. Adv Ther. 2011;28:51-61.

30. Baumgartner PC, Haynes RB, Hersberger KE, Arnet I. A systematic review of medication adherence thresholds dependent of clinical outcomes. Front Pharmacol. 2018;9:1290.

31. Boland MRS, van Boven JFM, Kruis $\mathrm{AL}$, et al. Investigating the association between medication adherence and health-related quality of life in COPD: methodological challenges when using a proxy measure of adherence. Respir Med. 2016;110: 34-45.

32. García-Sempere A, Hurtado I, Sanfélix-Genovés J, Rodríguez-Bernal C, Peiró S, Sanfélix-Gimeno G.
Improving the accuracy of medication adherence measures using linked prescription and dispensation data: findings from the ESOSVAL cohort of patients treated with osteoporosis drugs. Curr Med Res Opin. 2019;35:1535-44.

33. Karve S, Cleves MA, Helm M, Hudson TJ, West DS, Martin BC. Good and poor adherence: optimal cutpoint for adherence measures using administrative claims data. Curr Med Res Opin. 2009;25:2303-10.

34. Nicholas JA, Edwards NC, Edwards RA, Dellarole A, Grosso M, Phillips AL. Real-world adherence to, and persistence with, once- and twice-daily oral diseasemodifying drugs in patients with multiple sclerosis: a systematic review and meta-analysis. BMC Neurol. 2020;20:281.

35. Burks J, Marshall TS, Ye X. Adherence to diseasemodifying therapies and its impact on relapse, health resource utilization, and costs among patients with multiple sclerosis. Clinicoecon Outcomes Res. 2017;9:251-60.

36. Health Mart Systems I, Smart Retailing Rx. Star ratings: what pharmacists need to know. https:// join.healthmart.com/clinical-performance/starratings-what-pharmacists-need-to-know/. Accessed 28 Oct 2020. 